Acta Hispanica (2020) Supplementum II: 907-922

\title{
IDEARIO DE PODER SOCIOPOLÍTICO Y ACADÉMICO EN LA CREACIÓN DE LA UNIVERSIDAD Y SU INCIDENCIA EN LAS UNIVERSIDADES LATINOAMERICANAS
}

\author{
Amalfi Del Socorro Padilla Castilla - Ingrid Blanco Hernández \\ Universidad de Cartagena
}

\begin{abstract}
Resumen: Es pertinente iniciar este escrito desde la conceptualización y fundamentación de un plan de trabajo, del cual el método histórico, permitió la reflexión análisis y conceptualización del desarrollo, en la creación y desarrollo de las universidades en el mundo. Iniciando con el concepto de ideario, que representa el conjunto de ideas principales de los autores, que, en el caso en mención, hace referencia a la Universidad como un ente colectivo, de dinámica académica social y geopolítica. Desde esta instancia la Universidad no es posible estudiarla sin poner en consenso dichos procesos, los cuales dinamizan su quehacer y pertinencia en el territorio en el cual se gestan sus procesos socio académicos, administrativos y de gestión con la dinámica geopolítica y de poder del país. En la universidad no es posible historiografiar sus inicios y desarrollos sin determinar sus procesos académicos, administrativos y de gestión como una institución de poder político. Los studium generale, como se entendía la institución universitaria, era una organización que se regía bajo el principio de construir saberes y de captar conocimientos que vinieran desde los diferentes puntos cardinales, asegurando la construcción del saber desde la determinación de una forma de configurar un grupo de intelectuales que tenían el poder del conocimiento como fuente de su control social. Estos poderes produjeron tensiones en el espacio europeo medieval, porque al mantener los vínculos con los poderes reinantes, la universidad estaba subsumida por su estructura, en un ente que controlaba los escenarios de reproducción del saber. Así, los conocimientos que se engendraban desde la universidad eran regulados por el papado, elemento que revela el carácter político que ha tenido la universidad desde su misma concepción. De buen grado pensaron y con razón los poderes papal y seglar en cabeza del emperador, que crear universidades eran cuño y timbre de gloria, prestigio, poder y medio conducente para la formación de funcionarios que en su momento sirvieran a monarquías y otras formas de gobierno. El auge numérico y geográfico de la expansión universitaria, se acentúa en los siglos XIV y XV, (precisamente en el siglo XIV por desavenencias de un grupo de profesores de la Universidad de Oxford, se crea la de Cambridge). Posteriormente se crean las de Padua, Nápoles, Toulouse, Praga, Viena, Heidelberg y Colonia, que serán las únicas universidades del país. En esta centuria nacen las primeras universidades escocesas. Hacia mediados del siglo XV, las universidades habían cubierto los países de cultura latina. Generándose no solo por ubicación geográfica sino el fomento de una política de poder y de saber, que inciden en Latinoamérica.

Palabras clave: universidad, ideario sociopolítico y poder.
\end{abstract}

Abstract: It is pertinent to initiate this writing from the conceptualization and foundation of a work plan, of which the historical method, allowed the reflection analysis and conceptualization of development, in the creation and development of universities in the world. Starting with the concept of ideology, which represents the set of main ideas of the authors, which in the case mentioned, refers to the University as a collective entity, of social and geopolitical academic dynamics. From this instance, the University cannot study it without putting these processes in consensus, which boost

Acta Hispanica, Hungría, Supplementum II: 907-922, 2020, ISSN: 1416-7263 | 907 
Ideario de poder sociopolítico y académico en la creación de la universidad y su incidencia en las universidades latinoamericanas

their work and relevance in the territory in which their socio-academic, administrative and management processes are gestated with the geopolitical and power dynamics of the country. In the university it is not possible to historiography its beginnings and developments without determining its academic, administrative and management processes as an institution of political power. The studium generates, as the university institution understood, was an organization that was governed by the principle of building knowledge and capturing knowledge that came from different cardinal points, ensuring the construction of knowledge from the determination of a way to configure a group of intellectuals who had the power of knowledge as a source of their social control. These powers produced tensions in the medieval European space, because by maintaining links with the reigning powers, the university was subsumed by its structure, in an entity that controlled the knowledge reproduction scenarios. Thus, the knowledge that was generated from the university was regulated by the papacy, an element that reveals the political character that the university has had since its conception. They willingly and rightly thought the papal and secular powers in the head of the emperor, that creating universities were a wedge and timbre of glory, prestige, power and half conducive to the formation of officials who once served monarchies and other forms of government. The numerical and geographical boom of university expansion is accentuated in the fourteenth and fifteenth centuries, (precisely in the fourteenth century due to disagreements by a group of professors at the University of Oxford, Cambridge is created). Subsequently, those of Padua, Naples, Toulouse, Prague, Vienna, Heidelberg and Cologne are created, which will be the only universities in the country. The first Scottish universities are born in this century. By the middle of the 15th century, universities had covered the countries of Latin culture. Generating not only by geographical location but the promotion of a policy of power and knowledge, which affect Latin America.

Keywords: University, Power, Socio Political Ideology.

\subsection{Pensamiento e ideario político y de poder en las universidades en el mundo y América Latina}

El desarrollo histórico de la universidad ha estado orientado desde sus orígenes a una serie de nociones y conceptos sobre lo que ha sido su presencia y evolución en el contexto mundial, nacional y local. Entre los historiadores hay consenso en sostener la tesis, que las universidades brotaron de la atmósfera social y cultural de la sociedad europea occidental urbana de los siglos XI y XII en correspondencia con la estructura social existente. Aun así, las primeras manifestaciones de lo que había de constituirse en el siglo XII, en la tercera de dos instituciones transcendentes que regían la sociedad cristiana, han sido situadas en 1140 en la ciudad de Bolonia, en donde la universidad del mismo nombre sentó sus reales pensamientos en medio de los poderes trascendentales que eran el espiritual (Sacerdotum), representado en el papado y el temporal (Imperium) formalmente encarnado en la persona del Sacro Emperador Romano. A ellos, se agregó la erudición (Studium) y la universidad fue su expresión manifiesta. Para utilizar deliberadamente una frase anacrónica, esas tres instituciones; el poder divino, el poder sobre la tierra y el poder de la erudición, conformaron una especie de autoridad de orden superior sobre una comunidad que no se identificaba principalmente por sus fronteras geográficas, sino más bien por su unidad 
religiosa y su integración social en torno a la religiosidad. De esta manera, en la medida en que la cristiandad se tenía por universal, estas tres instituciones eran las expresiones de poder organizadas de esa universalidad (Neave, 2001: 51). La universidad desde su concepción ha sido una institución de poder. Los studium generale, como se entendía la institución universitaria, era una organización que se regían bajo el principio de construir saberes y de captar conocimientos que vinieran desde los diferentes puntos cardinales, asegurando la construcción del saber, como una forma de configurar un grupo de intelectuales que tenían el poder del conocimiento como fuente de su control social. Estos poderes produjeron tensiones en el espacio europeo medieval, porque al mantener los vínculos con los poderes reinantes, la universidad estaba subsumida por esa estructura, que controlaba los escenarios de reproducción del saber. Los conocimientos que se engendraban desde la universidad eran regulados por el papado, elemento que revela el carácter político que ha tenido la universidad desde su misma concepción. De buen grado pensaron, y con razón, los poderes papal y seglar en cabeza del emperador, que crear universidades eran cuño y timbre de gloria, prestigio, poder y medio conducente para la formación de funcionarios que en su momento sirvieran a monarquías y otras formas de gobierno.

Así, el auge numérico y geográfico de la expansión universitaria, se acentúa en los siglos XIV y XV, (precisamente en el siglo XIV por desavenencias de un grupo de profesores de la Universidad de Oxford, se crea la de Cambridge). Posteriormente se crean las de Padua, Nápoles, Toulouse, Praga, Viena, Heidelberg y Colonia, que serán las únicas universidades del país. En esta centuria nacen las primeras universidades escocesas. Hacia mediados del siglo XV, las universidades habían cubierto los países de cultura latina. Desde esta perspectiva, las universidades de Italia y París, son fruto de la iniciativa de soberanos y gobiernos, en virtud de motivaciones político-territoriales. De esta manera, el aumento del número de universidades a lo largo de los siglos XIV y XV redujo la circulación interterritorial de estudiantes viajeros, este fenómeno fue más notable entre los jóvenes menos acaudalados. La movilidad estudiantil se hizo intraterritorial y aunque el movimiento entre los reinos y sus universidades no cesó, quedó en gran medida limitado a los alumnos adinerados.

En efecto, el fortalecimiento del territorialismo o supremacía de la clase terrateniente y con ella la decadencia de la autoridad fundadora del orden superior del papa y el emperador trajeron como consecuencias las reformas y contrarreformas que llevaron a las universidades a mantenerse al margen. Por el contrario, en aquellos países en los que confluían las fronteras religiosas entre la iglesia universal y las iglesias reformadas, las universidades llegaron a ser instrumentos destinados a asegurar la ortodoxia o marcar la frontera entre las interpretaciones opuestas del credo. En realidad, una de las pruebas formales de una soberanía recién proclamada era el derecho de los gobernantes locales a formar universidades (Neave, 2001: 54). 
Ideario de poder sociopolítico y académico en la creación de la universidad y su incidencia en las universidades latinoamericanas

\subsection{Surgimiento de la universidad "moderna": Rusia, Francia y Alemania}

Los movimientos de la reforma protestante, la implementación de la imprenta en la Europa occidental, la circulación democrática de los libros y la lectura individual, marcaron el nuevo rumbo de la historia de las universidades europeas. Las reformas instrumentales constituidas por reorganizar la administración del Estado, se convierten en un instrumento de poder que establece el soporte de las nuevas estructuras políticas que convierten a las universidades en centros de poder, que reproducen saberes que se toman como pilares de las ideas políticas, socioeconómicas de la sociedad.

En las de Prusia, Wilhelm von Humboldt en 1807, después de la derrota sufrida en la batalla de Jena, y la creación de la Université Imperiale, encarada por Napoleón I entre 1806 y 1808, se estiman estos instrumentos como acontecimientos claves para el desarrollo de la universidad moderna. Este modelo de reformas organizativas del Estado, se considera decisivo para el desarrollo de las universidades o para una mejor estructuración de su quehacer académico, no sólo porque ofrecieron un modelo que luego sería ampliamente emulado en otras partes del mundo; sino, por establecer en primer lugar la relación entre la enseñanza y la investigación y en segundo lugar la relación entre las universidades y el Estado. Además, señalaron el fin de ese prolongado proceso que se había extendido durante dos siglos, como fue la incorporación final de la universidad como una institución de servicio público (Verger, 1986: 20).

Desde esta perspectiva, la universidad moderna reintroduce la ciencia y la investigación en el quehacer universitario. Y surge del modelo que Guillermo de Humboldt, quien definió a petición del emperador de Prusia, para la universidad de Berlín. Por otro lado, las ideas de Humboldt son el trasunto de una larga discusión alemana sobre la idea de universidad en la cual participaron sus pensadores más lúcidos.

En el caso de Alemania, se dio un fenómeno muy especial en la relación entre la universidad y el Estado, ya que el absolutismo de los monarcas impidió el desarrollo del espíritu indagador. Pero aun así es un ejemplo de los delicados medios de equilibrio que muchas veces adopta la relación universidad-Estado. Es decir, bajo el dominio de monarquías autocráticas y en el seno de universidades estatales carentes de autonomía fue posible un notable desarrollo intelectual, gracias al principio de la libertad académica, característico de las universidades alemanas.

En la Universidad de Halle, se destaca el magisterio de Christian Wolff, por la producción de un extraordinario desarrollo en casi todas las ramas científicas. En la Universidad de Gotinga se desenvuelve el sistema de enseñanza a través de seminarios, de gran importancia para el arraigo de la investigación científica. Mientras tanto, en la universidad de Jena, a principios del siglo XIX, se produjo una aglomeración de grandes intelectuales alemanes: Schiller, Schelling, Fichte, Hegel y Humboldt. Posteriormente, se funda la Universidad de Berlín (1810) según el esquema de Guillermo de Humboldt, el cual hace que la libertas philosophandi preconizada por Kant sea requisito indispensable para que la actividad académica, llegue a su madurez. Como se observa la estrecha relación 
entre docencia e investigación, influye luego en la organización de muchas otras universidades de diferentes partes del mundo y fue un elemento clave para el desarrollo científico que se produjo, a partir de entonces, en Alemania.

Bajo esta visión, la universidad alemana devino en una comunidad de investigadores que enseñaban haciendo uso principalmente de la mayéutica socrática; es decir, el aprendizaje se produce como resultado de la incorporación activa del estudiante en el quehacer científico, bajo la dirección de un maestro investigador (Tirso Mejía, 1981: 331).

Desde esta perspectiva, se puede afirmar que las reformas humboldtianas estaban al servicio de la idea de consolidar la innovación en la universidad, liberalizar el currículo y crear en el seno de la universidad las libertades formalmente reconocidas de enseñar y aprender. Además, concedieron libertad a las artes y las ciencias, sin mencionar la autonomía que otorgaban a la institución universitaria para determinar sus programas de investigación y enseñanza. En la interpretación humboldtiana y también en la concepción idealista de la universidad, en relación a la cultura, la ciencia y la enseñanza, estaban por encima del estado. Esta posición recuerda el concepto trascendente del Sacerdotum medieval, en donde la responsabilidad de la universidad era obrar como la expresión más elevada de la unidad cultural. Y, su independencia estaba respaldada por el marco legislativo que le suministraba el Estado. En contraste, el modelo napoleónico si bien atendía al principio de meritocracia en la vida nacional, también, debía garantizar la unidad política y la estabilidad de la nación, expresada como una unidad histórica y geográfica (Rohrs, citado por Tünnermann, 2005: 15).

\subsection{La universidad en Inglaterra}

La universidad inglesa considerada la más evolutiva de la época, se propone la educación general superior de sus alumnos en los varios órdenes de la vida. El primero de estos (teóricamente), su cultura y su educación en el conocimiento puro, meramente intelectual, es decir, una educación propiamente científica. Sin embargo, se da una variación en la relación entre el Estado y la Universidad donde las reformas de las dos antiguas Universidades como la de Oxford y Cambridge tuvieron mucho que ver con el surgimiento de la función pública profesional en oposición al servicio civil caracterizado por el clientelismo y mantenido por las prebendas políticas representadas en las reformas Northcote Trevelyan de 18541. En este sentido, en Inglaterra, la reforma de la

1 Las consecuencias de estas reformas no sólo han afectado al funcionamiento de la Administración, sino también a la relación entre ésta, el Gobierno y el Parlamento y, más concretamente, a la doctrina de la responsabilidad individual de los ministros, que es la convención constitucional que regula la relación entre estas tres instituciones en el sistema político británico. La mayoría de los autores coinciden en afirmar que de entre todas las reformas que se ponen en marcha desde finales de los setenta, la implantación de las recomendaciones del Programa Next Steps ha supuesto la transformación más importante de la Administración central británica desde el Informe Northcote-Trevelyan de 1854. Calificada como revolucionaria por algunos, lo cierto es 
Ideario de poder sociopolítico y académico en la creación de la universidad y su incidencia en las universidades latinoamericanas

administración pública y la de la universidad se desarrollaron en dos esferas separadas y el status de la universidad como una corporación autónoma y poseedora de sus propios bienes permaneció intacto. En cuanto al control administrativo, los vínculos entre la nación y la universidad, tomaron la forma muy específica de una jerarquía que descendía desde el ministerio a la universidad, encargada en la persona de un funcionario permanente comisionado para ejercer la auditoria dentro de cada establecimiento. Este funcionario, tenía las responsabilidades formales de actuar a nombre del gobierno, verificar los gastos y asegurarse que tanto los procedimientos como las estructuras en el ámbito de la universidad se ajustaran a las condiciones fijadas por el legislador vigente (director universitario), que en este caso encarnaban la presencia directa de la administración central en el seno de la universidad. En este sentido, la personificación de la responsabilidad pública y la rendición de cuentas, frente a ésta presencia erigida en símbolo de jerarquía ascendente, derivaba facultades de autoridad dentro de la universidad; estaba esa otra personificación con diversos grados de magnificencia en los títulos y esplendor en la toga, y en el rector o presidente de la universidad (Neave, 2001: 68-69).

Este esquema cronológico permite ubicarnos en el contexto de creación de las universidades, lo que dimensiona las características socio-académicos, políticos y culturales de los diferentes modelos universitarios implementados por las universidades europeas y norteamericanas. En cuanto a las universidades europeas se expresan tres tipos de modelos primarios como fueron: la Universidad Alemana de formación investigadora, la Universidad Británica y el que se denominaba latino bajo el cual se soslayaba el francés napoleónico y los modelos secundarios, como el ruso y el norteamericano. En relación al modelo de la universidad la francesa y su copia de la española, profesionalizadora" (De los Ríos, citado por Borrero Canaval, 2001: 31).

\subsection{La universidad en Norteamérica}

El modelo alemán fue imitado por las universidades norteamericanas, comenzando por la Universidad John Hopkins (1876) en Baltimore, la cual introdujo en Estados Unidos el modelo universitario Alemán basado en la estrecha asociación entre investigación y docencia, el cual se aplicaba en las facultades de ingeniería y programas de tecnologías, sin perjuicio de la existencia paralela de los institutos tecnológicos que se iniciaron como una universidad consagrada únicamente a los estudios de postgrado.

Una de las más antiguas universidades fundada en las colonias inglesas de Norteamérica fue la de Harvard, que nació con el nombre de Colegio de Harvard en

que su recomendación más sobresaliente - la creación de Agencias separadas pero dependientes del núcleo central de cada departamento ministerial- fue lo primero que provocó un profundo debate acerca de las consecuencias de esta medida para el mantenimiento de la ya debilitada doctrina de la responsabilidad individual de los ministros. 
honor a su primer benefactor y siguiendo el modelo del "residence college" inglés. Esta institución durante la primera etapa, se estableció en la costa oriental norteamericana y obedecían al espíritu que vivió Inglaterra en los siglos XVII y XVIII. Primero vinculadas a la iglesia y al orden social establecido, se fundaron algunas puritanas (Harvard, Yale); otras anglicanas (el Colegio de William and Mary en Columbia) o presbiterianas (Princeton). Su carácter distintivo fue su concentración en el nivel pregraduado, correspondiente a un programa de artes liberales de tipo clásico y destinado a la formación de los hijos de las nuevas clases dirigentes de la colonia (Tirso Mejía, 1981: 331).

La enseñanza puramente profesional se desarrolla en una etapa posterior, junto con la expansión del país hacia el oeste. Otra característica de la educación superior norteamericana fue la preeminencia de las universidades privadas, que generalmente cobraban altos aranceles, pero que también, sostenían importantes programas de becas y préstamos estudiantiles. Así mismo, la educación superior norteamericana es la que recibe mayores donaciones de fuentes privadas y públicas. También, es la que ha creado los vínculos más fuertes entre el sector empresarial y productivo. Pero el aporte más sobresaliente de la educación universitaria norteamericana en relación a la organización de las universidades en el mundo entero es, la introducción del departamento como una unidad académica básica, en sustitución de la cátedra aislada y unipersonal. Este germen de la departamentalización surgió en la Universidad de Harvard, cuando un profesor del Collège decidió impartir una sola asignatura y especializarse en ella en vez de asumir, como era usual hasta entonces, todas las llamadas artes liberales; había en Harvard un tipo de organización académica distinta, centrada en las asignaturas que más tarde serían los departamentos (Tünnermann, 2005: 37).

La introducción del sistema departamental no fue una simple innovación pedagógica. Hace más de cien años comenzaron los requerimientos productivos de ese país por personas especializadas cada vez más diversificados. El impacto sobre las universidades fue notable, obligando a la revisión de sus propios principios para poder subsistir. De esta manera, se hizo imprescindible eliminar programas anacrónicos o mediocres y tender hacia una orientación individualizada, organizar equipo de trabajo, este hecho trajo como consecuencia aumentar la dedicación de los docentes, para transmitir el saber, transformando la actividad de formación con la activa participación de todos los interesados. En forma simultánea, la diversificación de temas hizo necesaria la creación de subdivisiones organizativas que se denominaron departamentos y surgieron principalmente por iniciativa de cada disciplina o campo profesional y para satisfacción de los requerimientos de cada área especializada del saber. Por lo tanto, se constituyeron en verdaderos centros de convivencia y acción coordinada de especialistas dentro de cada universidad que tuviera suficientes conocimientos sobre el tema. Por otro lado, la excesiva departamentalización condujo a la compartimentalización basada en la unidisciplinariedad (Tünnermann, 2005: 38). El impacto de este hecho llevó al sociólogo alemán Hanns-Albert Steger a señalar el peligro de los departamentos en las universidades 
Ideario de poder sociopolítico y académico en la creación de la universidad y su incidencia en las universidades latinoamericanas

de los Estados Unidos. "El departament norteamericano [...] es en la práctica, un grupo de profesores que administran colectivamente un presupuesto comercial de la producción del conocimiento: así, se llega a la transformación interna de la universidad en una empresa comercial". Con todo esto, eminentes teóricos de las universidades latinoamericanas entre ellos el brasileño Darcy Ribeiro, sostiene que hasta ahora no se ha creado algo mejor para elevar los niveles académicos de las universidades y propiciar la investigación.

De esta manera, en Norteamérica se intensificó el número de universidades en los siglos XIX y XX, con una filosofía del desarrollo autónomo (Tünnermann, 2005: 39). Esta expansión universitaria fue un hecho planetario; casi todos los rincones del mundo tienen universidades que por causa de los colonialismos o de otros influjos, están investidas más o menos de notas distintas de los modelos clásicos. A estas universidades es lo que Ferrer denomina "modelos emergentes de universidad", el autor dice: "son estilos no clásicos de universidad, carecen de filósofos que la hayan precisado a satisfacción, luchan por su autonomía pero se consideran muy ligadas a la sociedad de la que emanan y a la cual desean moldear y conducir hacia un auténtico desarrollo, anhelan máximas libertades académicas, pero están aferradas al poder ascensional de los títulos" (citado por Múnera Ruiz, 2005: 36).

\subsection{La universidad republicana en América Latina}

En América Latina el surgimiento de la república no implicó la modificación de las estructuras socioeconómicas de la colonia. En este sentido, según Tünnermann, el movimiento de independencia careció de un contenido realmente revolucionario, limitándose en gran medida a la sustitución de autoridades peninsulares por los criollos, representantes de oligarquías terratenientes y de la naciente burguesía comercial (16-17). Los procesos de independencia en América Latina, más que configurar cambios estructurales y procesos de transformación radical con el pasado colonial, se conciben como un proceso de mimetización de elementos simbólicos, estructuras sociales y mecanismos de poder que se conjugan con las diversas prácticas reformistas que trajo los nuevos acontecimientos de la independencia. Lo importante aquí es mostrar que la dinámica de resignificación de las estructuras coloniales se desarrolló con la mirada puesta en los nuevos acontecimientos que se presentaban en la Europa ilustrada. Entonces, estos ideales de la ilustración y los nuevos aires de libertad intelectual sirvieron de modelos para configurar las instituciones del nuevo régimen, tomando elementos ideológicos de la revolución francesa como un dispositivo para configurar una nueva forma de mirar la universidad. Dentro de este panorama de admiración exaltado por la Ilustración francesa, no es extraña la adopción del modelo napoleónico de universidad para la república cuando se trató de reformar la universidad colonial. Sin embargo, las luchas de independencia no echaron paso atrás a la vida estática y rígida que llevaban estas instituciones en la postrimería del régimen colonial. Las universidades estuvieron al 
margen del movimiento independentista aun cuando muchas de ellas entraron al movimiento de la Ilustración y contribuyeron así mismo, a formar la conciencia independentista de algunos próceres principalmente los provenientes de las capas medias.

Algunas universidades lograron revitalizar su enseñanza a través de la introducción del método experimental y otras permanecieron fieles a un escolasticismo estático donde nada más se podía aportar el conocimiento. Por otro lado, la investigación abandonaría las anteriores aulas plenas de silogismo y buscaría posición en las academias llamadas "ciencias americanas", lo que contribuyó a sellar el destino de las universidades coloniales, puesto que al emigrar de ellas la ciencia, su suerte se definió con la república donde lograron las universidades definir su "función".

De esta manera, las universidades latinoamericanas en lugar de buscar la renovación por la brecha abierta de los sabios americanos, se constituían en una respuesta original, a la prevalencia de la investigación científica entre las nuevas generaciones. La república tras las pugnas que tuvieron lugar entre liberales y conservadores inmediatamente después de la independencia, encontró un mejor espacio que el modelo colonial, que había sido sustituido por el proyecto napoleónico de la universidad francesa a tono con las inquietudes intelectuales que se vivían. Lo anterior se enriqueció con la asimilación de la investigación científica, elemento fundamental del modelo humboldtiano de universidad.

En su estudio sobre la universidad latinoamericana, Ocampo anota que Darcy Ribeiro se interesó por la comparación universitaria con los modelos de las Universidades francesas, alemanas, inglesas, norteamericanas, soviética y japonesa. Cuando descubrió como alguna de sus estructuras universitarias habían sido imitadas en diversos aspectos por algunas universidades latinoamericanas. No obstante, el legado de la universidad francesa y alemana se desarrolló cuando éstas se convirtieron en agencias internacionales de integración nacional, de movilización cívica y de incorporación de sus sociedades a la civilización de su tiempo (Ocampo López, 2006: 146-148).

De esta manera, la universidad francesa transformó una sociedad arcaica, seccionada en provincias heterogéneas, aportando de manera significativa en la construcción de la sociedad moderna, unificada por la lengua, un cuerpo de comprensiones comunes, una actitud afirmativa hacia el mundo y hacia sí misma. Bajo esta consideración, se puede afirmar que el legado del modelo napoleónico que se instauró después de la revolución, fue el artífice de la creación de las universidades oficiales financiadas por el Estado, con un sistema de escuelas autónomas, encargadas de formar a los profesionales de las diversas disciplinas, especialmente en derecho, medicina, farmacia, letras y ciencias. Por otro lado, se encargó a la escuela politécnica, la formación de los cuadros técnicos y a la escuela normal superior, que actuarían como difusores en toda la nación de la nueva cultura erudita de base científica. El modelo de universidad napoleónica influyó decisivamente en la organización de las universidades oficiales de América Latina, que no imitaron la institución universitaria centralizadora, característica de la Francia imperial, 
Ideario de poder sociopolítico y académico en la creación de la universidad

y su incidencia en las universidades latinoamericanas

sino el ejemplo de universidades con un conglomerado de facultades y escuelas autárquicas, independientes y con una tendencia hacia el profesionalismo.

Desde la perspectiva política, la adopción del modelo napoleónico para las universidades, configuraba mentalidades que se constituían en dos pensamientos; dentro del utilitarismo, con el fin de lograr un desarrollo coherente en todos los aspectos políticos económicos demográficos humanitario, procedente de la misma filosofía (utilitarista) ya su evolución hacia el radicalismo democrático, bajo el pensamiento de Bertrand (2002: 105). Quien se mostraría luego partidario de un poder fuerte y bien armado para la acción. Además, sostuvo la teoría de la democracia pura, el sufragio universal como soberanía del pueblo, estricta subordinación de los gobernantes a los gobernados, ausencia de contrapesos y sistemas fuertemente centralizados. Estos factores de poder del modelo napoleónico, se manifiestan en el pensamiento administrativo de las universidades latinoamericanas que tienen como constitución el Estado. Para Napoleón, la aceptación de lo público y lo privado fue una tolerancia de algo contrario a los principios de la revolución no deseado, sino coyunturalmente. De esta manera, la coexistencia de lo oficial y lo privado, con lo cual tuvo que contar la legislación napoleónica, es nota predominante en la educación latinoamericana, y se constituye en sana coexistencia, que de acuerdo con los principios democráticos de libertad, debería concebirse contrario al pensamiento de Napoleón; que de hecho en no pocas legislaciones invierte el orden nacional y entrega al Estado la preeminencia de la educación (Bertrand, 2002: 44-48). Desde esta perspectiva, se derivan normas constitucionales o legales de quejumbrosa permisión estatal para la iniciativa privada. Según los antecedentes y relaciones señalados por Darcy Ribeiro, las universidades hispanoamericanas más antiguas surgieron en los primeros años de la conquista y colonización. Las Universidades de Salamanca y Alcalá de Henares, las dos universidades más famosas de la época fueron los modelos que inspiraron la creación de estas organizaciones en el nuevo mundo. Es decir, las universidades que se crearon fueron una réplica del modelo hispánico de enseñanza superior: señorial, escolástica y clerical.

Al finalizar el siglo XVI Hispanoamérica tenía seis universidades, elevándose a diecinueve en los años de independencia. En Colombia en realidad existieron dos períodos en el desarrollo de la universidad. El primer período se inició con la fundación de la Universidad de Santo Tomás en el siglo XVI de igual manera, durante el Nuevo Reino de Granada (siglo XVII) fue fundada la Universidad Javeriana de Santafé, la cual nunca gana el carácter de universidad pública, pese a la existencia del régimen de patronato y a los privilegios de las instituciones eclesiásticas, debido a su dirección autónoma que con frecuencia le confieren ciertas facultades a los claustros de profesores y además se aplican irrestrictamente las reglas exclusivas y discriminatorias que exigen limpieza de sangre, tradición católica, pertenencia de familia con títulos de nobleza y radical exclusión de indios, negros y gente de oficios viles o de color quebrado.

Desde el siglo XVII, la Real Audiencia de Santa Fe, formuló la necesidad de fundar la universidad pública, directamente dependiente de la monarquía, quebrando el monopolio ejercido por las comunidades religiosas. Esta tendencia se tornó al tomar auge el 
movimiento progresista de la ilustración, en la metrópoli a partir de la segunda mitad del siglo XVIII, además es alentada por la influencia de Campomanes y de Feijóo, mediante la penetración clandestina de la filosofía racionalista en las colonias americanas. Este pensamiento penetró hondamente en las nuevas generaciones en 1781 y se convierte en el rango histórico de pre- revolución de independencia, en las nuevas generaciones de aristocracia criolla de Popayán, Santafé, Tunja y en la burguesía de Cartagena heredada de la oligarquía de comerciantes que ejercía el monopolio legal sobre el comercio en la metrópoli (García Nossa, 1985: 43-44). Consecuente con el razonamiento anterior, se puede afirmar que la creación de las universidades en América Hispánica, tienen que ver con la alianza entre la iglesia representado por el Papa y el Estado en cabeza del Virrey, para el control y establecimiento de redes sociales territoriales que se extendieron por toda América (45).

El segundo período de gran importancia en el proceso de evolución y análisis de las relaciones de poder en las instituciones universitarias, fue el ocasionado con motivo de la independencia. Durante este período, denominado republicano, la universidad experimenta grandes cambios, a través de la organización y consolidación de las universidades oficiales alrededor del modelo napoleónico. Este modelo reorganizó la universidad en escuelas profesionales autárquicas, desprovistas de una estructura integradora. Además, estableció la cátedra como unidad operativa de la docencia e investigación en la enseñanza superior. Es decir, una institución con currículos estáticos y obligatorios para la formación profesional; una variedad de carreras ofrecidas a los estudiantes, sin correlación con las necesidades de la sociedad. De esta manera se institucionalizan las Universidades elitistas caracterizadas por una organización administrativa burocrática.

La imitación y el calco del modelo universitario francés, fue el camino escogido por la república para nacionalizar y modernizar las antiguas universidades coloniales, consideradas como vestigios medievales. A su vez, la universidad francesa acababa de experimentar profundos cambios bajo la égida de Napoleón, entre cuyos ideales educativos se mencionan el gran impulso a los politécnicos. Es decir, la concepción universitaria napoleónica se caracterizó por el énfasis profesionalista, determinaba la desarticulación de la enseñanza y la sustitución de la universidad por una suma de escuelas profesionales; como sustitución se materializó en la investigación científica, que dejó de ser tarea universitaria y pasa a otras instituciones académicas; hecho que llevó a las universidades a someterse a la tutela y guía del Estado, a cuyo servicio debe consagrar sus esfuerzos mediante la preparación de los profesionales. Por consiguiente, su misión es proveer adiestramiento cultural y profesional a la elite burguesa, imprimiéndole a la vez un particular sello intelectual, encaminado a promover la unidad y estabilidad política del Estado.

Además, es importante resaltar en América Latina dos modelos clásicos de la universidad nacional: la creada por Andrés Bello en Santiago de Chile en el año 1843 y la Universidad Nacional Autónoma de México, creada en el año 1910 fundada por Justo 
Ideario de poder sociopolítico y académico en la creación de la universidad

y su incidencia en las universidades latinoamericanas

Sierra. En cuanto a la Universidad Nacional de Chile Huneeus Madge opina que "era una universidad urbana y adecuada al siglo en su condicionalidad social" (1973, 23-45). Este mismo esquema, fue instaurado en Bolivia, pero fracasó puesto que existían en el contexto boliviano otras circunstancias sociales, diferentes estructuras políticas y económicas, enmarcadas en relaciones de Estado que no permitían la adopción de un modelo social de universidad, porque el poder lo detentaba los criollos ilustrados.

Sin embargo, el nuevo esquema que se institucionalizó desplazó en gran parte al clérigo como la figura central de la universidad en América Latina, sustituyéndolo por la persona con investidura de saber como es el caso del abogado, formado principalmente a través del Derecho Romano y del Código Civil, que Don Andrés Bello redactó para Chile, inspirado en el Código Francés y conocido igualmente como el Código Napoleónico. De esta manera Bello, asumió las más importantes funciones sociales y estructuró las nacientes repúblicas, constituyendo un producto típico de la universidad latinoamericana en el siglo XIX, puesto que la universidad colonial preparaba a los servidores de la iglesia para la educación, la ética y la moral, la república por su parte debía dar "idoneidad" a los funcionarios del Estado (Huneeus Madge, 1973: 33).

En México la universidad como institución surge con motivo del primer centenario de su independencia, fue don Justo Sierra, quien logró el nombre de Universidad Nacional de México. De esta manera ésta empieza a tener la tendencia hacia la culturalidad en el pueblo mexicano, elemento fundamental que dio lugar al reclamo por la autonomía. Sin embargo, solo hasta 1929, el presidente Emilio Portes decreta la autonomía universitaria, transformando la universidad mexicana en Universidad Nacional Autónoma de México. Esto permitió el posicionamiento de la universidad y lo más importante constituía el rescate de la identidad de un pueblo.

En este espacio de búsqueda de autonomía, se representa a través de dos conceptos que identifican este proceso: uno es el poder constituido, donde el pueblo o multitud se entrega en defensa convirtiéndose en expresión de diversidad y de la pluralidad de la existencia que siempre cuestiona lo que hace el Estado; esta expresión logra identificarse e interactuar con el otro concepto que es el poder constituyente donde la fuerza de la multitud es fuente y sujeto de lo político, dando paso a lo que se podría denominar el concepto de una crisis, las cuales se forman en busca de posicionamiento y poder. Desde esta visión, todo el poder político está en la multitud, que no ocupa el lugar del Estado, porque es deseo de lucha, de constitución, es fuerza que está por fuera del Estado, por eso no se somete, es democracia, por lo cual, en México, se da esta etapa evolutiva desde donde la universidad, logra su autonomía.

$\mathrm{El}$ análisis de este marco histórico permite conocer de donde surgen los esfuerzos por darle forma político-institucional a un Estado republicano. Este se inicia, por una parte con la reestructuración de la administración del Estado y por otra, la definición de un modelo liberal de educación superior, que se fue redefiniendo y transformándose a lo largo de medio siglo, con el surgimiento de grandes líneas o tendencias de orientación teórica ideológica entre las que se encuentran; un tipo de educación superior y enseñanza 
universitaria impartida en su totalidad por el Estado, con formación inmediata de los cuadros profesionales de la primera república.

De acuerdo a Tünnermann, la universidad napoleónica que surgió en América Latina, produjo los profesionales requeridos a las necesidades perentorias; igualmente, les correspondió a ellos completar la organización de las nuevas repúblicas y promover su progreso (2005: 68). Pero indudablemente el número de profesionales que logró ingresar a ellas no correspondió en cantidad, ni calidad a las reales necesidades de las regiones. Por otro lado, la universidad republicana tampoco logró ampliar la base social de la matrícula estudiantil, que siguió siendo representativa de las clases dominantes y poderosas. En este sentido, al permanecer intactas las estructuras fundamentales de la sociedad, perduró la naturaleza elitista de la institución hasta la primera mitad del siglo XX; donde se manifestaron mediante diferentes formas, la inconformidad por el sentido aristocrático de la universidad. Esta tradición educativa la compartía y usufructuaba una porción muy pequeña de la población y sus efectos no influían sobre la sociedad en general, sino que operaban a través de una elite.

De esta manera, la dinámica del proceso en la implementación del modelo educativo, ha sido particularmente intenso y rápido en Colombia. Y antes que empezaran los fenómenos que lo definieron como tal, la sociedad colombiana tenía una economía que se fundamentaba en la hacienda de baja intensidad y una población que era mayormente rural. Esto evidencia, como en 1938 el 71\% de su población vivía en áreas rurales y el $87 \%$ residía en ciudades menores de 200 mil habitantes (Tünnermann, 2005: 68). Esta concentración de la población en ciudades menores permite la apertura de unos esquemas democráticos y autónomos, frente a los procedimientos implementados en los espacios académicos y administrativos de la universidad.

Esta tradición educativa, la compartía y usufructuaba una porción muy pequeña de la población y sus efectos no influían sobre la sociedad en general, sino que operaban a través de grupos de poder. Estos aspectos muestran que la universidad ofreció oportunidades para estudiar una serie de carreras técnicas nuevas, pero no contempló como consecuencia de la matriz adoptada la posibilidad de cultivar las ciencias en sí mismas, aparte de sus aplicaciones inmediatas. Es así como por muchas décadas en Colombia fue posible estudiar ingeniería civil, medicina o farmacia, más no matemáticas, biologías o química. Así mismo, las condiciones sociopolíticas y económicas, del periodo en estudio determinaron los sistemas de masificación y modernización de los procesos académicos y administrativos en las universidades latinoamericanas. La naturaleza del proceso modernizador indujo una acelerada expansión de la matrícula escolar de los niveles primarios y secundario por una parte, como consecuencia de la necesidad de educación para el funcionamiento de la población en ambientes urbanos y por otra, como efecto de las urgentes demandas de la industria cuya complejidad tecnológica requería tanto obreros especializados como ingenieros de diferentes ramas y también de la demanda del Estado y la economía privada, cuya racionalización dio lugar al nacimiento y al vertiginoso crecimiento de la economía, la administración y la contaduría. Nacen de allí la expansión y la diversificación de la 
Ideario de poder sociopolítico y académico en la creación de la universidad y su incidencia en las universidades latinoamericanas

universidad e ingresan a ella nuevos grupos sociales que conformaran el grueso de la burocracia, tecnocracia y los profesionales liberales del país moderno.

La orientación de la pedagogía experimenta también cambios rápidos y sustanciales y el interés del alumno pasa a ser su centro. Se propugnan la creatividad, la crítica y el desarrollo de la imaginación. El maestro pasa de ser el poseedor del conocimiento a constituirse en coordinador de los intereses del estudiante. Esta es, por supuesto, la teoría que informa la escuela modernizante, aunque solamente en casos aislados se constituye en práctica real. El discurso pedagógico de los maestros, sin embargo, sigue esas líneas. Debido a la expansión del magisterio de todos los niveles como un grupo ocupacional cuantitativamente importante, que da pie a la aparición y a la vez unión de actores académicos. La rapidez de ese proceso, no obstante, significó en algunos momentos la improvisación en la formación de maestros en desmedro de su calidad. Una característica central del proceso de modernización en Colombia es la naturaleza desigual de su desarrollo. Por una parte la función homogeneizadora actuó sobre el marco cultural diverso según las distintas regiones del país, lo cual produjo una superposición e interacción de las distintas formas culturales al interior de la cultura de la modernización, afectando así la estructura y organización de la familia y la producción de la educación (Parra Sandoval, 1996: 66-67).

Sin embargo, estas instituciones universitarias latinoamericanas no han logrado cambios por sí solas, el siglo XIX y el XX son periodos de constante cambio y a ellas se les impone adaptarse a estas exigencias de esas nuevas dinámicas. Sumado a ello, la adopción espontánea de modelos académicos extranjeros, los cuales han sido asumidas en diferentes espacios y apropiadas en nociones y conceptos diferentes, ubicando América Latina en un contexto irreal y homogeneizado. En consecuencia, la universidad moderna es la que fortalece y enriquece el pensamiento universitario, en ella se establece el compromiso de enseñar, aprender e investigar. De esta manera, la reestructuración careció del sentido de afirmación nacional que se buscaba para las nuevas sociedades y siguió más bien el sendero de la alienación cultural que había caracterizado a los esfuerzos de renovación universitaria. Por otro lado, la temprana fundación de las universidades en América, conllevó a la intención de un "traspaso cultural", pero en ambos casos la respuesta careció de autenticidad, debido a que los ideales de cambio surgieron de las entrañas de la realidad americana y en nada correspondieron a las necesidades latinoamericanas. Además, estaban destinadas a las capas altas de la sociedad, lo que constituía una gran red de poder social y político (Borrero Canabal, 2001: 32).

En este orden de ideas, el proceso de industrialización nacional y el auge de la gran industria con capital multinacional que aparece en los años cuarenta, permitió un proceso de cambio en la sociedad colombiana conocido como modernización, que se caracterizó por fuertes cadenas migratorias del campo a la ciudad y del vertiginoso crecimiento de las ciudades; además estuvo acompañada por el surgimiento de las clases medias y urbanas; así, como un gran grueso del proletariado. Finalmente, surgen otros elementos como la explosión demográfica, el afianzamiento del Estado como racionalizador de la economía, a 
través de los procesos de planificación, como la aparición significativa de corrientes de movilidad social especialmente ligadas a la educación.

Por último, teniendo las universidades republicanas como referente el modelo francés, permitió un traspaso cultural, mediante la construcción de identidades, creando grupos de elites que permearon todas las instancias de poder mediante acciones decisorias en la consolidación de una nación de tipo partidista que da lugar a la fragmentación de la sociedad colombiana. Por otro lado, el acceso a las universidades, estaban destinadas a las clases poderosas, dominantes terratenientes y oligárquicas de la época.

Así, que en el contexto de la latinoamericana podemos localizar a la Universidad de Cartagena, creada bajo la égida napoleónica desde sus estructuras organizativas hasta la funcionalidad de quehacer, que está identificada y controlada por el Estado. Este recorrido genealógico de la historia de la universidad en el mundo, contextualizan la forma como las estructuras políticas e ideológicas dan como resultado la aparición y mantenimiento de la Universidad de Cartagena como un escenario de poder. La historia Universidad de Cartagena, es la reproducción del modelo político de las universidades latinoamericanas, cuyos poderes y estrategias se diseminan en las diferentes formas de estructuración cultural que cada uno de los contextos proponen.

Este capítulo intenta mostrar un panorama general que sirva de base teórica y conceptual para la construcción de la historia del poder en la universidad. Los modelos y las formas de organización han sido un retrato de una realidad general de la universidad latinoamericana, pero al trabajar la historia de la Universidad de Cartagena, nos damos cuenta de sus particularidades y sus formas de construir redes sociales de poder, a partir del enfrentamiento por el saber y los choques y las luchas por mantener en empoderamiento de cada uno de los grupos que la conforman después de la segunda mitad del siglo XX.

\section{Referencias bibliográficas}

Bertrand, Michel (comp.) (2002). Configuraciones y Redes de Poder. Un análisis de las relaciones sociales en América Latina. Caracas: Fondo Editorial Tropykos.

Borrero Canaval, Alfonso (2001). La Universidad y las Ciencias desde el renacimiento hasta 1800. En: Seminario Permanente Sobre la Universidad. Bogotá.

García Nossa, Antonio (1985). El ciclo de la dominación hispano-colonial. En: La Crisis de la Universidad. Bogotá: Editorial Plaza \& Janes. 43-44.

Huneeus Madge, Carlos (1973). La reforma en la Universidad de Chile: Santiago: Sala de Gráfica de CPU.

Múnera Ruiz, Leopoldo (2005). Poder. Trayectorias Teóricas de un Concepto. Colombia Internacional, 62. 32-49. 
Ideario de poder sociopolítico y académico en la creación de la universidad y su incidencia en las universidades latinoamericanas

Neave, Guy (2001). La dimensión europea en la enseñanza superior: incursión en el empleo moderno de las analogías históricas. En: Educación Superior: Historia y política. Barcelona: Gedisa.

Ocampo López, Javier (2006). Darcy Ribeiro: sus ideas educativas sobre la universidad y el proceso civilizatorio de América Latina. Revista Historia de la Educación Latinoamericana, 8. 137-160.

Parra Sandoval, Rodrigo (1996). La Universidad. Escuela y Modernidad en Colombia, Universidad y Proceso de Modernización. Colombia: Tercer Mundo Editores.

Tirso Mejía, Ricart (1981). La Universidad en la Historia Universal. Santo Domingo: Editorial de la Universidad Autónoma de Santo Domingo.

Tünnermann Bernheim, Carlos (2005). La Autonomia Universitaria frente al mundo Globalizado. Santo Domingo.

Verger, Jacques (comp.) (1986). Histoire des Universités en France. Toulouse: Privat. 